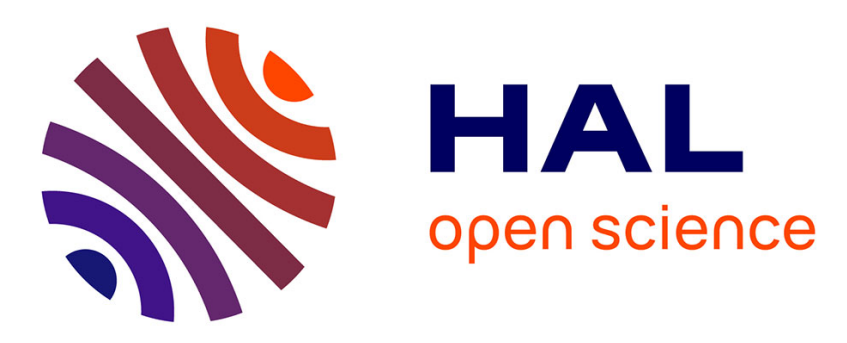

\title{
Universal Time-Symmetric Number-Conserving Cellular Automaton
}

\author{
Diego Maldonado, Andrés Moreira, Anahí Gajardo
}

\section{To cite this version:}

Diego Maldonado, Andrés Moreira, Anahí Gajardo. Universal Time-Symmetric Number-Conserving Cellular Automaton. 21st Workshop on Cellular Automata and Discrete Complex Systems (AUTOMATA), Jun 2015, Turku, Finland. pp.155-168, 10.1007/978-3-662-47221-7_12 . hal-01442471

\section{HAL Id: hal-01442471 \\ https://hal.inria.fr/hal-01442471}

Submitted on 20 Jan 2017

HAL is a multi-disciplinary open access archive for the deposit and dissemination of scientific research documents, whether they are published or not. The documents may come from teaching and research institutions in France or abroad, or from public or private research centers.
L'archive ouverte pluridisciplinaire HAL, est destinée au dépôt et à la diffusion de documents scientifiques de niveau recherche, publiés ou non, émanant des établissements d'enseignement et de recherche français ou étrangers, des laboratoires publics ou privés.

\section{(c)(1)}

Distributed under a Creative Commons Attribution| 4.0 International License 


\title{
Universal Time-symmetric Number-conserving Cellular Automaton *
}

\author{
Diego Maldonado ${ }^{1}$, Andrés Moreira ${ }^{2}$, and Anahí Gajardo ${ }^{1}$ \\ 1 Departamento de Ingeniería Matemática and Centro de Investigación en Ingeniería \\ Matemática, Universidad de Concepción, Casilla 160-C, Concepción, Chile \\ diegomaldonado@udec.cl \\ 2 Departamento de Informática, Universidad Técnica Federico Santa María, Vicuña \\ Mackenna 3939, Santiago, Chile
}

\begin{abstract}
We show the existence of Turing-universal and intrinsically universal cellular automata exhibiting both time symmetry and number conservation; this is achieved by providing a way to simulate reversible $\mathrm{CA}$ with time-symmetric $\mathrm{CA}$, which preserves the number-conserving property. We also provide some additional results and observations concerning the simulation relations between reversible, time-symmetric and number-conserving $\mathrm{CA}$ in the context of partitioned $\mathrm{CA}$.
\end{abstract}

\section{Introduction}

Reversibility is an important property in the realm of cellular automata, both because of its obvious theoretical consequences and because of the most practical applications for massive distributed computing models where heat dissipation would be unwanted. It has therefore been well studied (see reviews in $[5,9]$ ), and some of this effort has been directed towards showing computational capabilities (by showing the existence of universal reversible CA, under different notions of universality) and towards the easy construction of reversible CA.

A more specific kind of reversibility, which is common in physical theories, is that of time symmetry, and it has been only recently applied to cellular automata [4]. In time-symmetric systems there is an involution (i.e., a root-ofidentity transformation) that reverses the flux of time, so that further application of the system's dynamics has the same effect as going back with the inverse transformation, if the involution had not been applied. Time symmetry is a natural property for study, because of its ubiquity in physical theories, and also because of potential practical concerns: one of the advantages of using reversible $\mathrm{CA}$ for distributed computation, besides heat minimization, is that we may actually reverse time ocassionally, if we want to find the source of a phenomenon or debug a computation mistake. In that case, using the same CA (and, in a practical setting, the same circuits) to move backwards in time would be certainly handy.

\footnotetext{
* This work was partially supported by CONICYT-Chile through BASAL project CMM, Universidad de Chile, and FONDECYT project\# 1140833.
} 
A further CA property that has attracted some attention is number conservation: here the CA states are assumed to be numbers, and their sum over finite -or periodic- configurations is preserved. The inspiration comes again from physics, with its conservation laws, and from models of simple interacting agents, when there is a fixed number of them. Number conservation can be seen as the most simple case of more general additive conservation laws in cellular automata (moreover, whatever is true for number-conserving CA usually carries on nicely to the more general settings).

Despite apparently being a rather restrictive property, number conservation allows the existence of intrinsically universal CA (roughly speaking, CA that can simulate any other) and hence also of CA which are Turing-universal [7]. This is also the case for time symmetry [4], with the caveat that intrinsic universality is restricted to the simulation of all other reversible $\mathrm{CA}$. This implies the existence of full computational capabilities and of arbitrarily complex phenomena within these classes. In this manuscript we show that this is also true for the CA that share both properties. This is done in Section 3, by giving a general simulation procedure that turns arbitrary reversible CA into time-symmetric ones while preserving number conservation. The existence of reversible and number-conserving Turing-universal CA was already proved by Morita [10].

While mapping the territory we came across a possible path to the same result, which was not ultimately fruitful, but which yielded a few results and observations that are presented here in Section 4. The main result is a generalization of one from Morita [10], showing that any reversible partitioned CA can be simulated by a reversible number-conserving $\mathrm{CA}$. We also define "intrinsic time symmetry" (ITS) in a natural way for partitioned CA, characterize ITS partitioned CA in terms of their local function, and show that the number-conserving CA simulating them (obtained through the generalized Morita construction) are time-symmetric. This could have been a path to the result of Section 3 if we had an example of intrinsic universality in ITS partitioned CA, which we currently do not. Nevertheless, the generalization does bridge two CA classes through a simulation relation. As a byproduct, it also gives a way to construct time-symmetric (number-conserving) CA by starting with ITS partitioned CA, which are rather easy to construct, and where ITS is a decidable property.

\section{Definitions}

Definition 1 (Cellular automata). $A$ 1-dimensional cellular automaton $(C A)$ $A$ is a 4-tuple $A=(\mathbb{Z}, Q, N, f)$ where $Q$ is a finite set called the state set, $N=\left(n_{1}, \ldots n_{m}\right) \in \mathbb{Z}^{m}$ is the neighborhood, and $f: Q^{m} \rightarrow Q$ is the local transition function.

$A C A$ defines a dynamics on the space $Q^{\mathbb{Z}}$ of configurations through the global transition function

$$
\begin{gathered}
F: Q^{\mathbb{Z}} \longrightarrow Q^{\mathbb{Z}}, \\
F(\alpha)_{i}=f\left(\alpha_{i+n_{1}}, \alpha_{i+n_{2}}, \ldots, \alpha_{i+n_{m}}\right) .
\end{gathered}
$$


The size of the neighborhood is $m$ and when $N=(-r,-r+1, . ., 0, . ., r)$, we say that it is a symmetric neighborhood of radius $r$. If $r=0$, the $C A$ is autarkic. When $n_{j} \geq 0$ for every $j$, the $C A$ is one way. $C A$ with the asymmetric neighborhood $(0,1)$ are said to have radius $1 / 2$.

Additive conservation laws occur in CA when some locally computed numerical attribute, added over periodic configurations, is preserved through time. The simplest version happens when the states themselves are numbers, and their sum is conserved. Here we give the definition based on finite configurations, which are configurations where all the cells, except for a finite number, are equal to 0 . This has been shown to be equivalent to the definition based on periodic configurations.

Definition 2 (Number conservation). Given a $C A A=(\mathbb{Z},\{0,1, \ldots, s\}, N, f)$, we say that it is number-conserving (NCCA) if for every finite configuration $c$

$$
\sum_{i \in \mathbb{Z}} F(c)_{i}=\sum_{i \in \mathbb{Z}} c_{i}
$$

Remark 1. The class of number-conserving CA is closed for composition and inverse.

Remark 2. In NCCA all states are quiescent, i.e., all homogeneous configurations are fixed points.

Definition 3 (Time symmetry). Given a reversible $C A A=(\mathbb{Z}, Q, N, f)$, we say that it is time-symmetric (TS) if there exists a CA with global transition function $H$ such that

$$
H \circ F \circ H=F^{-1} \quad \wedge \quad H \circ H=I d .
$$

$H$ in this case is an involution associated to the time-symmetric $C A A$.

Remark 3. A direct consequence of time symmetry is that

$$
\forall n \in \mathbb{Z}, H \circ F^{n} \circ H=F^{-n} .
$$

Remark 4. The class of time symmetry CA is closed for inverse.

Working with reversible $\mathrm{CA}$, and in particular constructing them, is a bit cumbersome since identifying reversibility from the local transition function is not direct, and only an exponential algorithm exists. This has led a number of researchers to prefer the more convenient formalism provided by partitioned cellular automata. They are a particular case of CA, but they are general in the sense that every CA can be simulated by a partitioned CA.

Definition 4 (Partitioned Cellular Automaton). A partitioned cellular automaton (PCA) is a 4-tuple $P=\left(\mathbb{Z}, Q=Q_{n_{1}} \times \ldots \times Q_{n_{m}},\left(n_{1}, \ldots, n_{m}\right), f\right)$, where $Q_{i}$ are finite sets and $f: Q \rightarrow Q$ is the local transition function of $P$.

The global transition function of $P$ is $F: Q^{\mathbb{Z}} \rightarrow Q^{\mathbb{Z}}$ given by

$$
F(\alpha)_{i}=f\left(\alpha_{i+n_{1}}\left(n_{1}\right), \ldots, \alpha_{i+n_{m}}\left(n_{m}\right)\right)
$$

where $\alpha \in Q^{\mathbb{Z}}$, and $\alpha_{i}(j)$ is the coordinate indexed by $j$ of the $i$-th cell in $\alpha$. 
It is practical to view PCA as the composition of two CA: a product of shifts and an autarkic CA.

Proposition 1. Given a PCA $P=\left(\mathbb{Z}, Q=Q_{n_{1}} \times \ldots \times Q_{n_{m}},\left(n_{1}, \ldots, n_{m}\right), f\right)$, then its transition function $F$ is equal to $A_{f} \circ I_{\left(n_{1}, \ldots, n_{m}\right)}$, where

- $A_{f}$ is the autarkic $C A$ defined by $f: A_{f}(\alpha)_{i}=f\left(\alpha_{i}\right)$,

$-I_{\left(n_{1}, \ldots, n_{m}\right)}=\sigma^{n_{1}} \times \sigma^{n_{2}} \times \ldots \times \sigma^{n_{m}}:\left(Q_{n_{1}} \times \ldots \times Q_{n_{m}}\right)^{\mathbb{Z}} \rightarrow\left(Q_{n_{1}} \times \ldots \times Q_{n_{m}}\right)^{\mathbb{Z}}$,

- $\sigma$ is the shift of configurations and $\forall n \in \mathbb{Z}, \forall i \in \mathbb{Z}, \sigma^{n}(x)_{i}=x_{i+n}$.

Definition 5 (Universality). In order to prevent unnecessary clutter here, we will dispense with the formal definitions of universality. Turing-universality is common lore by now, and as for intrinsic universality, we refer the reader to [2] for definitions as well as excelent discussion. However, intrinsic universally depends on the precise simulation relation being used, and it is therefore important to remark that the simulations presented here comply with the definition of "injective bulking" introduced in [2], and that is the sense in which intrinsic universality must be understood.

\section{Universality}

In [4] arbitrary reversible CA are transformed into time-symmetric CA by using the fact that the product $\mathrm{CA} F \times F^{-1}$ simulates $F$ and is time-symmetric. If the original $\mathrm{CA}$ is number-conserving, the product $\mathrm{CA}$ does not have to be, since there is no natural relabeling of its states (which are ordered pairs) that may ensure number conservation. On the other hand, one of the ideas used by Morita [10] to transform a reversible PCA into a number-conserving CA is to represent vectors through numbers over a larger set. Here we combine these two ideas to transform a reversible number-conserving $\mathrm{CA}$ into a time-symmetric number-conserving CA.

Theorem 1. Given a reversible number-conserving CA there exists a time-symmetric number-conserving $C A$ that simulates it.

Proof. Let $A=(\mathbb{Z}, Q=\{0,1, \ldots, s-1\}, N, f)$ be a reversible and number conserving CA. Let us define first the automaton $\bar{A}=(\mathbb{Z}, \bar{Q}, N, \bar{f})$ where $\bar{Q}=\{k s$ : $k=0,1, \ldots, s-1\}$ and $\bar{f}\left(\bar{q}_{1}, \ldots, \bar{q}_{|N|}\right)=s f\left(\bar{q}_{1} / s, \ldots, \bar{q}_{|N|} / s\right)$. It is just $A$, but with the states multiplied by $s$. Now let $Q^{\prime}$ be $Q^{\prime}=\left\{0,1, \ldots, s^{2}-1\right\}$; clearly, for every $q^{\prime} \in Q^{\prime}$ there is a unique pair $(q, \bar{q}) \in Q \times \bar{Q}$ such that $q^{\prime}=q+\bar{q}$. We can thus define two projections $p: Q^{\prime} \rightarrow Q$ and $\bar{p}: Q^{\prime} \rightarrow \bar{Q}$ such that for every $q^{\prime} \in Q^{\prime}$, $q^{\prime}=p\left(q^{\prime}\right)+\bar{p}\left(q^{\prime}\right)$. Let $P$ and $\bar{P}$ be the natural generalizations of these functions to $Q^{\prime \mathbb{Z}}$. Our time-symmetric number-conserving $\mathrm{CA} A^{\prime}=\left(\mathbb{Z}, Q^{\prime}, N, f^{\prime}\right)$ is defined through the global transition function

$$
F^{\prime}(\alpha)=F(P(\alpha))+\bar{F}^{-1}(\bar{P}(\alpha))
$$


Such a cellular automaton exists because $F$ is reversible, $\bar{F}$ is conjugated to $F$, and because the addition of CAs is also a CA, by the Hedlund-LyndonCurtis theorem. Clearly, $F^{\prime}$ simulates $F$, because $Q \subset Q^{\prime}$, and if $\alpha \in Q^{\mathbb{Z}}$ then $F^{\prime}(\alpha)=F(\alpha)+\bar{F}^{-1}(0)=F(\alpha)$. Let us prove now that $F^{\prime}$ has the requested properties.

Reversibility. The inverse of $F^{\prime}$ is given by $F^{\prime-1}(\alpha)=F^{-1}(P(\alpha))+\bar{F}(\bar{P}(\alpha))$ :

$$
\begin{aligned}
F^{\prime-1}\left(F^{\prime}(\alpha)\right) & =F^{\prime-1}(\underbrace{F(P(\alpha))}_{\in Q}+\underbrace{\bar{F}^{-1}(\bar{P}(\alpha))}_{\in \bar{Q}}) \\
& =F^{-1}(F(P(\alpha)))+\bar{F}\left(\bar{F}^{-1}(\bar{P}(\alpha))\right. \\
& =P(\alpha)+\bar{P}(\alpha) \\
& =\alpha .
\end{aligned}
$$

Number conservation. Let $\alpha \in Q^{\prime \mathbb{Z}}$ be a finite configuration. Since both $F$ and $\bar{F}$ are number-conserving,

$$
\begin{aligned}
\sum_{i \in \mathbb{Z}} F^{\prime}(\alpha)_{i} & =\sum_{i \in \mathbb{Z}}\left(F(P(\alpha))_{i}+\bar{F}^{-1}(\bar{P}(\alpha))_{i}\right) \\
& =\sum_{i \in \mathbb{Z}} F(P(\alpha))_{i}+\sum_{i \in \mathbb{Z}} \bar{F}^{-1}(\bar{P}(\alpha))_{i} \\
& =\sum_{i \in \mathbb{Z}} P(\alpha)_{i}+\sum_{i \in \mathbb{Z}} \bar{P}(\alpha)_{i} \\
& =\sum_{i \in \mathbb{Z}}\left(P(\alpha)_{i}+\bar{P}(\alpha)_{i}\right) \\
& =\sum_{i \in \mathbb{Z}} \alpha_{i}
\end{aligned}
$$

Time symmetry. The involution $H$ that makes $F^{\prime}$ time-symmetric is defined by $H(\alpha)=\underbrace{s \cdot P(\alpha)}_{\in \bar{Q}}+\underbrace{\frac{1}{s} \cdot \bar{P}(\alpha)}_{\in Q}$. It is an autarkic CA, and $H \circ H=I$. Let us prove that $H \circ F^{\prime} \circ H=F^{\prime-1}$,

$$
\begin{aligned}
H\left(F^{\prime}(H(\alpha))\right) & =H\left(F\left(\frac{1}{s} \cdot \bar{P}(\alpha)\right)+\bar{F}^{-1}(s \cdot P(\alpha))\right) \\
& =s F\left(\frac{1}{s} \cdot \bar{P}(\alpha)\right)+\frac{1}{s} \bar{F}^{-1}(s \cdot P(\alpha)) \\
& =s \frac{1}{s} \bar{F}(\bar{P}(\alpha))+\frac{1}{s} s F^{-1}(P(\alpha)) \\
& =F^{\prime-1}(\alpha)
\end{aligned}
$$


Corollary 1. There exists a Turing-universal time-symmetric and number-conserving $C A$.

Proof. This follows from the existence of a Turing-universal reversible numberconserving CA, established by Morita [10] based on several of his previous results.

Corollary 2. There exists an intrinsically-universal time-symmetric and number-conserving $C A$ within the class of reversible $C A$.

Proof. Durand-Lose [3] proves the existence of a reversible intrinsically universal PCA of radius 1. On the other hand, Morita shows in [8] that any RPCA of radius 1 can be simulated by a one-way RPCA of radius $1 / 2$, and in [10] that any one-way RPCA of radius $1 / 2$ can be simulated by a reversible NCCA. Along with the present theorem, these results imply the existence of a time-symmetric intrinsically universal NCCA.

\section{The road not taken}

In [10], in order to obtain a Turing-universal reversible NCCA with a small neighborhood, Morita developed a way to transform a one way PCA of radius $1 / 2$ into a number-conserving $\mathrm{CA}$ of radius $3 / 2$ (i.e. neighborhood $(-1,0,1,2)$ ). His CA is not time-symmetric and it could not have been, since time symmetry in a one way PCA implies equicontinuity (see Proposition in the Appendix), which in turns implies periodicity. Nevertheless, his construction can be generalized to PCA of arbitrary neighborhood, and as we shall see, it does preserve time symmetry if it is present (in a sense to be defined) in the original PCA.

Proposition 2. Given a reversible PCA, there exists a reversible and numberconserving $C A$ that simulates it.

Proof. Let $P$ be a reversible PCA with state sets $Q_{i}=\left\{q_{i 0}, q_{i 1}, \ldots, q_{i\left(\left|Q_{i}\right|-1\right)}\right\}$; without loss of generality, and only for the sake of simplifying the proof, its neighborhood can be assumed to have the form $(-n, \ldots, n)$.

We follow the idea of Morita's construction in [10], which is to duplicate each set of states $Q_{i}$ into two sets that we call here $\hat{S}_{i} \subseteq \mathbb{N} \cup\{0\}$ and $\breve{S}_{i} \subseteq \mathbb{N} \cup\{0\}$, so that for each $q_{i j}$ there exist $\hat{s}_{i j} \in \hat{S}_{i}$ and $\check{s}_{i j} \in \check{S}_{i}$ satisfying that $\hat{s}_{i j}+\check{s}_{i j}$ is a constant that depends only on $i$. In order to compute this numbers we define two bijective funcions for each $i$ : $\hat{\phi}_{i}$ and $\check{\phi}_{i}$, which also ensure that the $\check{s}_{i}$ and $\hat{s}_{i}$ of different $i$ are on different scales and can be added into a single number wihout losing information.

The $k$-th cell of a configuration is represented by two cells in the number conserving CA, indexed by $2 k$ and $2 k+1$. In this way, the sum of the $2 k$-th and $2 k+1$-th cells in the representing configuration will be equal to a constant $K$, independent from the content of the original cell. Now we formally define all of the needed sets and functions, for each $i \in\{-n, . ., n\}$ : 
$-K_{-n-1}=1, K_{i}=\prod_{j=-n}^{i} 2\left|Q_{j}\right|$

$-\hat{S}_{i}=\left\{k K_{i-1}: k=0, \ldots,\left|Q_{i}\right|-1\right\}$

- $\check{S}_{i}=\left\{\left(k+\left|Q_{i}\right|\right) K_{i-1}: k=0, \ldots,\left|Q_{i}\right|-1\right\}$

$-\hat{\phi}_{i}: Q_{i} \rightarrow \hat{S}_{i}, \hat{\phi}_{i}\left(q_{i k}\right)=k K_{i-1}$

- $\check{\phi}_{i}: Q_{i} \rightarrow \check{S}_{i}, \check{\phi}_{i}\left(q_{i k}\right)=\left(2\left|Q_{i}\right|-k-1\right) K_{i-1}$

- $\hat{S}=\sum_{i=-n}^{n} \hat{S}_{i}, \breve{S}=\sum_{i=-n}^{n} \breve{S}_{i}$

$-S_{i}=\hat{S}_{i} \cup \check{S}_{i}, S=\sum_{i=-n}^{n} S_{i}$

$-\hat{\phi}: Q \rightarrow \hat{S}, \hat{\phi}\left(a_{-n}, \ldots, a_{n}\right)=\sum_{j=-n}^{n} \hat{\phi}_{j}\left(a_{j}\right)$

$-\check{\phi}: Q \rightarrow \check{S}, \check{\phi}\left(a_{-n}, \ldots, a_{n}\right)=\sum_{j=-n}^{n} \check{\phi}_{j}\left(a_{j}\right)$

With these definitions it is easy to see that

- there are projections $p_{i}: S \rightarrow S_{i}$ such that for $s \in S, s=\sum_{i=-n}^{n} p_{i}(s)$;

$-\left|Q_{i}\right|=\left|\hat{S}_{i}\right|=\left|\check{S}_{i}\right|=\frac{\left|S_{i}\right|}{2}$;

$-\forall i, \forall j, \hat{\phi}_{i}\left(q_{i j}\right)+\check{\phi}_{i}\left(q_{i j}\right)=K_{i}-K_{i-1}$; and

$-K_{n}=|S|$.

Each cell of $P$ is transformed into two cells of the new automaton by an injective but not surjective function $\Psi: Q^{\mathbb{Z}} \rightarrow S^{\mathbb{Z}}$, defined as follows.

$$
\Psi(\alpha)_{i}= \begin{cases}\hat{\phi}\left(\alpha_{i / 2}\right) & \text { if } i \text { is even } \\ \check{\phi}\left(\alpha_{(i-1) / 2}\right) & \text { if } i \text { is odd }\end{cases}
$$

It is not difficult to define an $\bar{F}: S^{\mathbb{Z}} \rightarrow S^{\mathbb{Z}}$ such that $\Psi \circ F=\bar{F} \circ \Psi$ over the set $\Psi\left(Q^{\mathbb{Z}}\right)$; however, we need it to be number-conserving and reversible over the whole set $S^{\mathbb{Z}}$. This will be done by considering a decomposition $\bar{F}=\overline{A_{f}} \circ \bar{I}$ (akin to that of Proposition 1) which makes the diagram below commute, and choosing $\overline{A_{f}}$ and $\bar{I}$ in a way that ensures reversibility and number conservation in each of them.

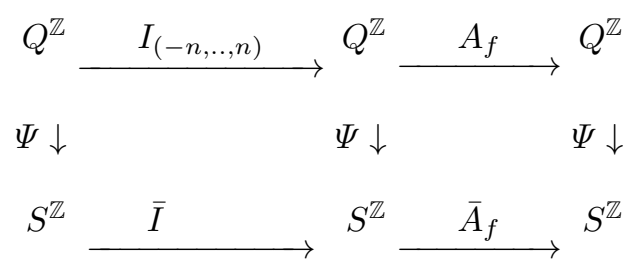

$\bar{I}: S^{\mathbb{Z}} \rightarrow S^{\mathbb{Z}}$ is just the equivalent of $I_{(-n, . ., n)}$ :

$$
\bar{I}(\alpha)_{i}=\sum_{j=-n}^{n} p_{j}\left(\alpha_{i+2 j}\right) .
$$

When $\Psi$ duplicates information into pairs of cells, we can distinguish between the left member (L) and the right member (R) of each pair, and moreover, these can be readily identified in configurations obtained through $\Psi$. In arbitrary 
configurations there can be wrong cells which are neither left nor right. Formally, given a configuration $\alpha \in S^{\mathbb{Z}}$, we define $L(\alpha), R(\alpha), W(\alpha) \subseteq \mathbb{Z}$ as follows:

$$
\begin{aligned}
i \in L(\alpha) & \Leftrightarrow \forall j \in\{-n, . ., n\}, p_{j}\left(\alpha_{i}\right) \in \hat{S}_{j} \wedge p_{j}\left(\alpha_{i}\right)+p_{j}\left(\alpha_{i+1}\right)=K_{j}-K_{j-1}, \\
i \in R(\alpha) & \Leftrightarrow \forall j \in\{-n, . ., n\}, p_{j}\left(\alpha_{i}\right) \in \check{S}_{j} \wedge p_{j}\left(\alpha_{i-1}\right)+p_{j}\left(\alpha_{i}\right)=K_{j}-K_{j-1}, \\
i \in W(\alpha) & \Leftrightarrow i \notin L(\alpha) \wedge i \notin R(\alpha) .
\end{aligned}
$$

The function $\bar{A}_{f}: S^{\mathbb{Z}} \rightarrow S^{\mathbb{Z}}$ is then defined by

$$
\bar{A}_{f}(\alpha)_{i}=\left\{\begin{array}{cl}
\hat{\phi} \circ f \circ \hat{\phi}^{-1}\left(\alpha_{i}\right) & \text { if } i \in L(\alpha) \\
\check{\phi} \circ f \circ \check{\phi}^{-1}\left(\alpha_{i}\right) & \text { if } i \in R(\alpha) \\
\alpha_{i} & \text { if } i \in W(\alpha)
\end{array}\right.
$$

Let us remark that $\bar{A}_{f}$ is not autarkic, but of radius 1 , since the neighbors of a cell must be known in order to determine its class. It is clear that $\bar{I}$ is reversible and number-conserving. To show that $\bar{A}_{f}$ has the same properties, we need to follow Morita and prove first that it preserves the sets $L, R$ and $W$.

To see this, first we notice that $L$ and $R$ cells come in pairs: if $i \in L(\alpha)$, then for every $j$ we have $p_{j}\left(\alpha_{i+1}\right)=K_{j}-K_{j-1}-p_{j}\left(\alpha_{i}\right)$ and $p_{j}\left(\alpha_{i+1}\right) \in \breve{S}_{j}$, and hence $i+1 \in R(\alpha)$; the converse is analogous. Any configuration consists therefore of blocks of $L-R$ pairs, possibly separated by cells in $W$. Moreover, when $i \in L(\alpha)$, we have $\hat{\phi}^{-1}\left(\alpha_{i}\right)=\check{\phi}^{-1}\left(\alpha_{i+1}\right)$. Therefore, when $\bar{A}_{f}$ is applied, $f\left(\hat{\phi}^{-1}\left(\alpha_{i}\right)\right)=f\left(\check{\phi}^{-1}\left(\alpha_{i+1}\right)\right), \bar{A}_{f}(\alpha)_{i} \in \hat{S}$ and $\bar{A}_{f}(\alpha)_{i+1} \in \check{S}$. This implies that $p_{j}\left(\bar{A}_{f}(\alpha)_{i}\right)+p_{j}\left(\bar{A}_{f}(\alpha)_{i+1}\right)=K_{j}-K_{j-1}$, for each $j$, and thus $i \in L\left(\bar{A}_{f}(\alpha)\right)$ and $i+1 \in R\left(\bar{A}_{f}(\alpha)\right)$. In other words, pairs in $\alpha$ are still pairs in $\bar{A}_{f}(\alpha)$, and neither cell can form a pair with a cell that was in $W$; so, $W$ is preserved too.

From this fact we can see that $\bar{A}_{f}$ is number-conserving, since cells in $W$ are not modified and the sum of the pairs is constant, equal to $|S|-1$. Reversibility is deduced from this too, since a bijective function is applied to each cell. Its inverse is expressed as follows.

$$
{\overline{A_{f}}}^{-1}(\alpha)_{i}=\left\{\begin{array}{cl}
\hat{\phi} \circ f^{-1} \circ \hat{\phi}^{-1}\left(\alpha_{i}\right) & \text { if } i \in L(\alpha) \\
\grave{\phi} \circ f^{-1} \circ \check{\phi}^{-1}\left(\alpha_{i}\right) & \text { if } i \in R(\alpha) \\
\alpha_{i} & \text { if } i \in W(\alpha)
\end{array}\right.
$$

We conclude that $\bar{F}=\bar{A}_{f} \circ \bar{I}$ is also reversible and number-conserving.

Since PCA are CA, the general definition of time symmetry applies to them: a PCA $F$ is time-symmetric if there is an involution $H$ such that $F \circ H=H \circ F^{-1}$. Notice, however, that $H$ does not need to be a PCA; in fact, neither does $F^{-1}$. We introduce a more convenient restricted notion of time symmetry, which requires $H$ to be a PCA with the same neighborhood of $F$.

Definition 6. Given a reversible $P C A P=\left(\mathbb{Z}, Q=Q_{n_{1}} \times \ldots \times Q_{n_{m}},\left(n_{1}, \ldots, n_{m}\right), f\right)$ we say that it is intrinsically time-symmetric (ITS) if there exists a PCA $H=$ $\left(\mathbb{Z}, Q=Q_{n_{1}} \times \ldots \times Q_{n_{m}},\left(n_{1}, \ldots, n_{m}\right), h\right)$ such that

$$
H \circ F \circ H=F^{-1} \quad \wedge \quad H \circ H=I d .
$$


Proposition 3. A PCA $P$ is intrinsically time-symmetric if and only if its neighborhood $\left(n_{1}, \ldots, n_{m}\right)$ is symmetric, and there exists a family of functions $h_{i}: Q_{i} \rightarrow Q_{-i}$ such that

$-h_{j}=h_{m+1-j}^{-1}$, and

$-h=\left(h_{1}, \ldots, h_{m}\right)$ satisfies $f \circ h=h \circ f^{-1}$.

Proof. As a first and also main step, we will prove that a PCA $H=(\mathbb{Z}, Q=$ $\left.Q_{n_{1}} \times \ldots \times Q_{n_{m}},\left(n_{1}, \ldots, n_{m}\right), h\right)$ is an involution if and only if its neighborhood is symmetric, the components $h_{j}$ of $h=\left(h_{1}, \ldots, h_{m}\right)$ depend only of their $(m+1-j)$ th coordinate, and they verify $h_{j}=h_{m+1-j}^{-1}$.

Suppose that $H$ is an involution and let $h_{j}$ be the components of $h$, so that $h=\left(h_{1}, \ldots, h_{m}\right)$. Notice that, a priori, each $h_{j}$ is a function with the whole set $Q$ as its domain. Let $\left(g_{j}: Q \rightarrow Q_{j}\right)_{j=1}^{m}$ be a family of functions such that $h^{-1}=g=\left(g_{1}, \ldots, g_{m}\right)$. Let $H=A_{h} \circ I$ be the decomposition of $H$ as per Proposition 1; recall that here $I=I_{\left(n_{1}, \ldots, n_{m}\right)}$ is a product of shifts. Since $H$ is an involution we have

$$
\begin{aligned}
I \circ A_{h} \circ I & =A_{h}^{-1}=A_{h^{-1}} \\
\forall i \in \mathbb{Z}, & \left(I \circ A_{h} \circ I(\alpha)\right)_{i}=\left(g_{1}\left(\alpha_{i}\right), \ldots, g_{j}\left(\alpha_{i}\right), \ldots, g_{m}\left(\alpha_{i}\right)\right) \\
\left(h_{1}\left(I(\alpha)_{i+n_{1}}\right), \ldots h_{j}\left(I(\alpha)_{i+n_{j}}\right), \ldots h_{m}\left(I(\alpha)_{i+n_{m}}\right)\right) & =\left(g_{1}\left(\alpha_{i}\right), \ldots, g_{j}\left(\alpha_{i}\right), \ldots, g_{m}\left(\alpha_{i}\right)\right)
\end{aligned}
$$

We obtain that $g_{j}\left(\alpha_{i}\right)=h_{j}\left(I(\alpha)_{i+n_{j}}\right)$, for each $j \in\{1, \ldots, m\}$. However, if we consider the decomposition of $\alpha_{i}$ as $\left(\alpha_{i}(1), \ldots, \alpha_{i}(m)\right)$, what we actually have is $g_{j}\left(\alpha_{i}(1), \ldots, \alpha_{i}(k), \ldots, \alpha_{i}(m)\right)=h_{j}\left(\alpha_{i+n_{j}+n_{1}}(1), \ldots, \alpha_{i+n_{j}+n_{k}}(k), \ldots, \alpha_{i+n_{j}+n_{m}}(m)\right)$

These two maps are equal and they depend on different sets of variables. They are not constant (since the global function is bijective), and must therefore really depend only on the variables they have in common. There is at most one of these for each $j$, and requires that for some $k$ we have $n_{j}+n_{k}=0$. This implies that the neighborhood must be symmetric, $g_{j}=h_{j}$ depends only on the $(m+1-j)$-th variable, and $\left|Q_{j}\right|=\left|Q_{m+1-j}\right|$.

Now using that $g=h^{-1}$, we obtain that $h_{j}^{-1}=g_{m+1-j}=h_{m+1-j}$.

The converse is trivial: if $h$ verifies the properties described above, it is easy to show that indeed $h=h^{-1}$, and $H=H^{-1}$.

The only part of the proposition which remains to be proved is the time symmetry relation verified at the local level. By using the decomposition of Proposition 1, let us write the global transition functions of the PCA $P$ and of its PCA involution as $F=A_{f} \circ I_{\left(n_{1}, \ldots, n_{m}\right)}$ and $H=A_{h} \circ I_{\left(n_{1}, \ldots, n_{m}\right)}$ respectively. The time symmetry of $F$ by way of $H$ is equivalent to the next equations:

$$
\begin{aligned}
H \circ F \circ H & =F^{-1} \\
\left(A_{h} \circ I\right) \circ\left(A_{f} \circ I\right) \circ\left(A_{h} \circ I\right) & =I^{-1} \circ A_{f}^{-1} \\
\left(I \circ A_{h} \circ I\right) \circ A_{f} \circ\left(I \circ A_{h} \circ I\right) & =A_{f}^{-1} \\
A_{h} \circ A_{f} \circ A_{h} & =A_{f}^{-1}=A_{f^{-1}}
\end{aligned}
$$


Since $A_{f}, A_{f^{-1}}$ and $A_{h}$ are autarkic, this last equation is equivalent to

$$
h \circ f \circ h=f^{-1} .
$$

It was shown in [4] that every reversible autarkic CA is time-symmetric, that is, for every bijective function $f: Q \rightarrow Q$ there exists some involution $h: Q \rightarrow Q$ such that $h \circ f \circ h=f^{-1}$. Proposition 3 imposes additional conditions to $h$, which will not be always verified. Thus, not every reversible $f$ defines an ITS PCA. For example, no involution $h$ on $Q=\{0,1\} \times\{0\} \times\{0,1\}$ satisfies $h \circ f \circ h=f^{-1}$ for the function $f$ that traverses the cycle $((0,0,1)(1,0,0)(1,0,1)(0,0,0))$.

Proposition 4. If $F$ is the transition function of an ITS PCA, then the numberconserving function $\bar{F}$ produced by Proposition 2 is time-symmetric.

Proof. Let $H$ be the involution that makes $F$ time-symmetric; we can suppose without loss of generality that the neighborhood is $\{-n, \ldots, n\}$. The involution that will work for $\bar{F}$ acts just like $H$ on $S$, ignoring whether the coordinates are in $\hat{S}_{j}$ or $\breve{S}_{j}$. For this we define the signature of a number $s \in S$ as the sets to which its projections belong, as follows.

$$
\begin{aligned}
\mathcal{C}: S & \rightarrow\{\wedge, \vee\}^{\{-n, \ldots, n\}} \\
\mathcal{C}(s) & =\left(c_{-n}(s), \ldots, c_{n}(s)\right) \\
\forall j \in\{-n, \ldots, n\}, c_{j}(s) & =\left\{\begin{array}{l}
\wedge \text { if } p_{j}(s) \in \hat{S}_{j} \\
\vee \text { if } p_{j}(s) \in \breve{S}_{j}
\end{array}\right.
\end{aligned}
$$

For convenience we define $S_{j}^{\vee}=\check{S}_{j}, S_{j}^{\wedge}=\hat{S}_{j}, \phi_{j}^{\vee}=\check{\phi}_{j}$ and $\phi_{j}^{\wedge}=\hat{\phi}_{j}$. Now $\tilde{A}_{h}$ is defined through the function $h$ by using the functions $\phi^{c}: Q \rightarrow \sum_{j=-n}^{n} S_{j}^{c_{j}}$, defined by $\phi^{c}(q)=\sum_{j=-n}^{n} \phi_{j}^{c_{j}}\left(q_{j}\right)$ to transform states to numbers with signature $c$ and vice versa. We will use the notation $\overleftarrow{c}=\left(c_{n}, . ., c_{-n}\right)$

$$
\begin{aligned}
\tilde{A_{h}}: S^{\mathbb{Z}} & \rightarrow S^{\mathbb{Z}} \\
\tilde{A}_{h}(\alpha)_{i} & =\overleftarrow{\phi^{\mathcal{C}\left(\alpha_{i}\right)}}\left(h\left(\left(\phi^{\mathcal{C}\left(\alpha_{i}\right)}\right)^{-1}\left(\alpha_{i}\right)\right)\right)
\end{aligned}
$$

$\tilde{A}_{h}$ is autarkic and it is an involution. Let us see that the associated automaton $\bar{H}=\tilde{A}_{h} \circ \bar{I}$ is also an involution, with $\bar{I}$ taken from Proposition 2.

$$
\begin{aligned}
\left(\tilde{A}_{h} \circ \bar{I}(\alpha)\right)_{i} & =\phi^{\overleftarrow{\mathcal{C}\left(\bar{I}\left(\alpha_{i}\right)\right)}}\left(h\left(\left(\phi^{\mathcal{C}\left(\bar{I}\left(\alpha_{i}\right)\right)}\right)^{-1}\left(\bar{I}\left(\alpha_{i}\right)\right)\right)\right) \\
& =\sum_{j=-n}^{n} \phi_{j}^{c_{-j}\left(\bar{I}\left(\alpha_{i}\right)\right)}\left(h_{j}\left(\left(\phi^{\mathcal{C}\left(\bar{I}\left(\alpha_{i}\right)\right)}\right)^{-1}\left(\sum_{j=-n}^{n} p_{j}\left(\alpha_{i+2 j}\right)\right)\right)\right)
\end{aligned}
$$


We use Proposition 3.

$$
=\sum_{j=-n}^{n} \phi_{j}^{c_{-j}\left(\alpha_{i-2 j}\right)}\left(h_{j}\left(\left(\left(\phi^{c_{-j}\left(\alpha_{i-2 j}\right)}\right)_{-j}^{-1}\left(p_{-j}\left(\alpha_{i-2 j}\right)\right)\right)\right)\right)
$$

We apply $\bar{I}$ from the left.

$$
\begin{aligned}
\left(\bar{I} \circ \tilde{A}_{h} \circ \bar{I}(\alpha)\right)_{i} & =\sum_{j=-n}^{n} p_{j}\left(\tilde{A}_{h} \circ \bar{I}(\alpha)_{i+2 j}\right) \\
& =\sum_{j=-n}^{n} \phi_{j}^{c_{-j}\left(\alpha_{i+2 j-2 j}\right)}\left(h_{j}\left(\left(\phi^{c_{-j}\left(\alpha_{i+2 j-2 j}\right)}\right)_{-j}^{-1}\left(p_{-j}\left(\alpha_{i+2 j-2 j}\right)\right)\right)\right) \\
& =\sum_{j=-n}^{n} \phi_{j}^{c_{-j}\left(\alpha_{i}\right)}\left(h_{j}\left(\left(\phi^{c_{-j}\left(\alpha_{i}\right)}\right)_{-j}^{-1}\left(p_{-j}\left(\alpha_{i}\right)\right)\right)\right) \\
& =\sum_{j=-n}^{n} \phi_{j}^{c_{-j}\left(\alpha_{i}\right)}\left(h_{j}\left(\left(\phi^{\mathcal{C}\left(\alpha_{i}\right)}\right)_{-j}^{-1}\left(\alpha_{i}\right)\right)\right) \\
& =\tilde{A}_{h}(\alpha)_{i}
\end{aligned}
$$

Now we just need to prove that $\bar{H} \circ \bar{F} \circ \bar{H}=\bar{F}^{-1}$. As we saw in the proof of the previous proposition, all we need to show is that $\tilde{A}_{h} \circ \bar{A}_{f} \circ \tilde{A}_{h}=\left(\bar{A}_{f}\right)^{-1}$. We analyze by cases depending on the class of cell $i$, and using the fact that if $s \in \hat{S}($ or $\check{S})$ then $\phi^{\mathcal{C}(s)}(s)=\hat{\phi}(s)\left(\phi^{\mathcal{C}(s)}(s)=\check{\phi}(s)\right)$.

Case 1: $i \in L(\alpha)$.

$$
\begin{aligned}
\tilde{A_{h}} \circ \overline{A_{f}} \circ \tilde{A_{h}}(\alpha)_{i} & =\hat{\phi} \circ h \circ \hat{\phi}^{-1} \circ \hat{\phi} \circ f \circ \hat{\phi}^{-1} \circ \hat{\phi} \circ h \circ \hat{\phi}^{-1}\left(\alpha_{i}\right) \\
& =\hat{\phi} \circ h \circ f \circ h \circ \hat{\phi}^{-1}\left(\alpha_{i}\right) \\
& =\hat{\phi} \circ f^{-1} \circ \hat{\phi}^{-1}\left(\alpha_{i}\right) \\
& =\bar{A}_{f-1}(\alpha)_{i} \\
& =\bar{A}_{f}^{-1}(\alpha)_{i}
\end{aligned}
$$

Case 2: $i \in R(\alpha)$. It is analogous to Case 1 .

Case 3: $i \in W(\alpha)$. We remark first that $\tilde{A}_{h}(\alpha)_{i} \in W$. Thus $\bar{A}_{f}\left(\tilde{A}_{h}(\alpha)_{i}\right)=$

$\tilde{A}_{h}(\alpha)_{i}$. Since $\tilde{A}_{h}$ is autarkic, $\left(\tilde{A}_{h}\left(\bar{A}_{f}\left(\tilde{A}_{h}(\alpha)\right)\right)\right)_{i}=\left(\tilde{A}_{h}\left(\tilde{A}_{h}(\alpha)\right)\right)_{i}=\alpha_{i}=$ $\left(\bar{A}_{f}\right)^{-1}(\alpha)_{i}$.

\section{Final remarks}

The road described in Section 4 could have led to the corollaries of Section 3, provided that universal ITS PCA had been known to exist. Let us briefly discuss the situation for each kind of universality. 
Is there an ITS Turing-universal PCA? Probably. In 1989 Morita and Harao [11] showed a way to simulate any given reversible Turing machine with a PCA of radius 1 . We currently conjecture that the construction (perhaps with slight modifications) yields an ITS PCA whenever the Turing machine is itself "time-symmetric". Since there has been little discussion of time symmetry in Turing machines, the notion used in this case is the simple one given in [1], where the involution consists of an autarkic CA involution on the tape, along with an independent permutation of the machine's state set. The Turing machine needs to be expressed in quadruples in order to give sense to this notion, since only in this case does its inverse transition function correspond to a Turing machine. With this definition, universal time-symmetric Turing machines do exist: following the technique of Kari and Ollinger [6], in order to obtain one, it is enough to join the inverse and the direct machine into one.

Is there an ITS intrinsically universal PCA? The intrinsically universal PCA built by Durand-Lose [3] is not ITS, but we believe that it could be modified to make it comply. In any case, its construction is quite complex and requires many states. Even if the modification worked, the existence of a simple intrinsically universal ITS PCA (or indeed, time-symmetric CA) remains an open question.

Finally, please notice that Section 4 provides a path for the construction of time-symmetric CA (possibly with the added bonus of number conservation), of which there are not that many. Besides this, the alternatives previously known to us were 1) to find involutions and blindly compose them, or 2) to design reversible PCA with a prescribed behavior (something for which PCA are quite useful), turn it into a reversible CA, and convert this into a time-symmetric CA. The new option is to construct an ITS PCA and then use Proposition 2 to get a time-symmetric CA. The ITS PCA could be designed by hand, or we could construct reversible PCA and test for the existence of an appropriate involution using Proposition 3, which is an improvement with respect to the general 1D CA case, where time symmetry is conjectured to be undecidable.

\section{References}

1. Julien Cassaigne, Nicolas Ollinger, and Rodrigo Torres-Avilés. A Small Minimal Aperiodic Reversible Turing Machine. https://hal.inria.fr/hal-00975244, 2014.

2. Marianne Delorme, Jacques Mazoyer, Nicolas Ollinger, and Guillaume Theyssier. Bulking II: classifications of cellular automata. Theoretical Computer Science, 412(30):3881-3095, 2011.

3. Jérôme Durand-Lose. Intrinsic universality of a 1-dimensional reversible cellular automaton. In Rüdiger Reischuk and Michel Morvan, editors, STACS, volume 1200 of Lecture Notes in Computer Science, pages 439-450. Springer, 1997.

4. Anahí Gajardo, Jarkko Kari, and Andrés Moreira. On time-symmetry in cellular automata. Journal of Computer and System Sciences, 78(4):1115-1126, 2012.

5. Jarkko Kari. Reversible cellular automata. In Clelia De Felice and Antonio Restivo, editors, Developments in Language Theory, volume 3572 of Lecture Notes in Computer Science, pages 57-68. Springer Berlin Heidelberg, 2005. 
6. Jarkko Kari and Nicolas Ollinger. Periodicity and immortality in reversible computing. In E. Ochmanski and J. Tyszkiewicz, editors, Mathematical Foundations of Computer Science (MFCS 2008), volume 5162 of LNCS, pages 419-430, 2008.

7. Andrés Moreira. Universality and decidability of number-conserving cellular automata. Theoretical Computer Science, 292(3):711-721, 2003.

8. Kenichi Morita. Computation-universality of one-dimensional one-way reversible cellular automata. Information Processing Letters, 42(6):325-329, 1992.

9. Kenichi Morita. Reversible computing and cellular automata: A survey. Theoretical Computer Science, 395(1):101 - 131, 2008.

10. Kenichi Morita. Universality of one-dimensional reversible and number-conserving cellular automata. In Enrico Formenti, editor, AUTOMATA JAC, volume 90 of EPTCS, pages 142-150, 2012.

11. Kenichi Morita and Masateru Harao. Computation-universality of one-dimensional reversible (injective) cellular automata. The Transactions of the IEICE, 72(6):758$762,1989$.

\section{$6 \quad$ Appendix}

Definition 7 (Equicontinuity). A CA with transition function $F$ is equicontinous if for every $N$ there exists $M \in \mathbb{N}$ such that $\alpha_{[-M, M]}=\beta_{[-M, M]}$ implies that for every $t \in \mathbb{N}, F^{t}(\alpha)_{[-N, N]}=F^{t}(\beta)_{[-N, N]}$.

Definition 8 ( $N$-sensitivity). A configuration $\alpha$ is said to be $N$-sensitive if for every $M \in \mathbb{N}$ there exists $\beta$ and $t \in \mathbb{N}$ such that $\alpha_{[-M, M]}=\beta_{[-M, M]}$ and $F^{t}(\alpha)_{[-N, N]} \neq F^{t}(\beta)_{[-N, N]}$.

It is known that a CA is equicontinous if and only if none of its configurations is $N$-sensitive, for any $N \in \mathbb{N}$.

Proposition 5. A one way time-symmetric PCA must be equicontinuous.

Proof. Let us first remark that one way CA can be time-symmetric without being periodic, even if the inverse is also one way in the same direction; therefore, our proof strongly relies on the partitioned nature of the CA.

Let $P$ be a time-symmetric $\mathrm{PCA}$ which is one way in the right direction, i.e. , its neighborhood $\left(n_{1}, . ., n_{m}\right)$ contains only non-negative coordinates, with $n_{m}$ being the biggest one.

Remark 5. A particular feature of reversible PCA is that when computing the preimage of a configuration, the content of a given cell determines, without ambiguity, part of the contents of the $m$ cells in its neighborhood. Which conversely implies that if one cell $i$ is perturbated, at least one cell in $\left\{i-n_{1}, . ., i-n_{m}\right\}$ will 'see' the difference in the next iteration of $P$.

Let us suppose that $P$ is time-symmetric with an involution $H$ of radius $r$, and that it is not equicontinuous. Let $\alpha$ be an $N$-sensitive configuration, and $\beta$ a configuration given by the definition for $M=N+2 r$. Furthermore, let $t$ be the least one for which $F^{t}(\alpha)_{[-N, N]} \neq F^{t}(\beta)_{[-N, N]}$. Since $P$ is one way, we 
can assure that the difference between these two last configurations lies between $N-n_{m}$ and $N$. Moreover, we can assume that $\alpha_{[-\infty, M]}=\beta_{[-\infty, M]}$, because differences to the left of $-M$ will not perturbate cells in $[-N, N]$.

From time symmetry, we have that $H(\beta)=F^{t}\left(H\left(F^{t}(\beta)\right)\right)$. But

$$
\begin{aligned}
H(\beta)_{[-\infty, N+r]} & =H(\alpha)_{[-\infty, N+r]}, \text { and } \\
H\left(F^{t}(\beta)\right)_{\left[N-n_{m}-r, N+r\right]} & \neq H\left(F^{t}(\alpha)\right)_{\left[N-n_{m}-r, N+r\right]}
\end{aligned}
$$

and using Remark 5, we obtain that

$$
\begin{aligned}
F^{t}\left(H\left(F^{t}(\beta)\right)\right)_{\left[N-n_{m}-r-t n_{m}, N+r\right]} & \neq F^{t}\left(H\left(F^{t}(\alpha)\right)\right)_{\left[N-n_{m}-r-t n_{m}, N+r\right]}, \text { i.e. } \\
H(\beta)_{\left[N-n_{m}-r-t n_{m}, N+r\right]} & \neq H(\alpha)_{\left[N-n_{m}-r-t n_{m}, N+r\right]},
\end{aligned}
$$

which is a contradiction. 